M. Miyake

Nagoya Math. J.

Vol. 117 (1990), 207-225

\title{
SOLVABILITY OF SYSTEMS OF ORDINARY DIFFERENTIAL EQUATIONS IN THE SPACE OF ARONSZAJN AND THE DETERMINANT OVER THE WEYL ALGEBRA
}

\author{
MASATAKE MIYAKE
}

\section{$\S 0$. Introduction}

N. Aronszajn introduced in [4] an abstract Frechét space $\mathscr{A}_{R}(0<R \leq \infty)$, which is isomorphic to the space of analytic solutions of the heat equation in $\boldsymbol{C}_{z}^{n} \times\{t \in \boldsymbol{C} ;|t-R|<R\}$ if $0<R<\infty$, and in $\boldsymbol{C}_{z}^{n} \times\{t \in \boldsymbol{C} ; \operatorname{Re} t>0\}$ if $R=\infty$, and called it the space of traces of analytic solutions of the heat equation. Hereafter, we call it the space of traces, shortly. Among other properties, it was shown that the space of tempered distributions $\mathscr{S}^{\prime}\left(\boldsymbol{R}^{n}\right)$, as well as the space of analytic functionals $\mathscr{H}^{\prime}\left(C^{n}\right)$, is identified with a dense subspace of the space of traces by the formula,

$$
\tilde{f}(z, t)=\langle f(x), e(z-x, t)\rangle \quad \text { for } f(x) \in \mathscr{S}^{\prime}\left(\boldsymbol{R}^{n}\right),
$$

where $e(z, t)$ is the heat kernel,

$$
e(z, t)=\frac{1}{(4 \pi t)^{n / 2}} \exp \left(-\frac{z^{2}}{4 t}\right), \quad z \in C^{n}, \quad \operatorname{Re} t>0 .
$$

Stimulating by his results, M.S. Baouendi [5] studied the space of traces from a different point of view. As an application, he proved the solvability of linear partial differential equations with constant coefficients in the space of traces. Moreover, he proved the solvability of the Mizohata equation in the space of traces,

$$
\frac{\partial u}{\partial x}+i x \frac{\partial u}{\partial y}=f
$$

which is solvable neither in the space of distributions nor in the space of hyperfunctions.

Received March 17, 1989.

*) This work was partially supported by Grant-in-Aid for Scientific Research (No. $63540109)$, Ministry of Education, Japan. 
He conjectured, in conclusion, that almost all linear partial differential equations with polynomial coefficients will be solvable in the space of traces.

This paper is concerned with his conjecture in the case of ordinary differential equations. More precisely, we shall prove an index theorem for systems of ordinary differential equations with polynomial coefficients and related results. To state our results, it is convenient and is essential to use the determinant associated with the Bernstein filtration for matrices of differential operators with polynomial coefficients. We have to note that our results have deep correspondences with those in the space of hyperfunctions, where the results are stated in terms of the determinant associated with the filtration by the order of differentiation.

In Section 1, we shall state our results and compare them with those in the space of hyperfunctions. We shall give a short summary of the space of traces in Section 2 developed by Baouendi [5], and we shall see that systems of equations in the space of traces are translated to systems of differential equations in the space of holomorphic functions, which enable us to employ the results in Miyake [9, 10]. Moreover, we shall give the proofs of results in Section 2 under assuming fundamental proposition (Proposition 2.3), which will be proved in Section 5. In Section 3 , we shall calculate a matrix of differential operators associated with a differential operator with polynomial coefficients. For the proof of the fundamental proposition, we shall give a brief summary of the determinant theory over the filtered rings developed by K. Adjamagbo [1] and others, in Section 4.

At the end of this section, the author would like to express his heartfelt thanks to Professor T. Matsuzawa for his suggestion of the problem and useful discussion.

\section{$\S 1$. Statement of results}

For $0<R \leq \infty$, we put

$$
D_{R}:=\{t \in C ;|t-R|<R\} \quad(0<R<\infty), \quad D_{\infty}:=\{t \in C ; \operatorname{Re} t>0\} .
$$

Let $H:=\partial / \partial t-\partial^{2} / \partial z^{2}$ be the heat operator, and define

$$
\operatorname{Sol}(H)_{R}=\left\{\tilde{f}(z, t) \in \mathscr{H}\left(C_{z} \times D_{R}\right) ; H \tilde{f}=0\right\},
$$

where $\mathscr{H}(\Omega)$ denotes the set of holomorphic functions in a domain $\Omega \subset C^{N}$ 
equipped with the uniform convergence topology on every compact set in $\Omega$. As mentioned In the Introduction, the space of traces of Aronszajn $\mathscr{A}_{R}(0<R \leq \infty)$ is an abstract Frechét space isomorphic to $\operatorname{Sol}(H)_{R}$,

$$
\mathscr{A}_{R} \cong \operatorname{Sol}(H)_{R}, \quad(0<R \leq \infty) .
$$

We denote by $W(\boldsymbol{C})$ the Weyl algebra of dimension 1 over $\boldsymbol{C}$, that is, the set of ordinary differential operators with polynomial coefficients,

$$
W(C):=\left\{P=\sum_{m+n<\infty} a_{m n} x^{m} D^{n} ; a_{m n} \in C\right\}, \quad D=d / d x .
$$

For an operator $P \in W(C)$, we denote by $\sigma_{w}(P)$ its Bernstein symbol, that is, for

$$
P=\sum_{m+n \leqq l} a_{m n} x^{m} D^{n}, \quad \text { where } a_{m n} \neq 0 \text { for some } m+n=l
$$

we define

$$
\sigma_{w}(P):=\sum_{m+n=l} a_{m n} x^{m} \xi^{n} \in \overline{C[x, \xi]},
$$

where $\overline{C[x, \xi]}$ denotes the set of homogeneous polynomials in $x$ and $\xi$. We denote by $M_{N}(W(C))$ the set of $N \times N$ matrices with entries in $W(C)$. Since the polynomial ring $C[x, \xi]$ is a unique factorization ring, we know by Adjamagbo [1] that for a matrix $P(x, D) \in M_{N}(W(C))$ we can define the determinant $\operatorname{det}_{w}(P) \in \overline{C[x, \xi]}$ of $P$ associated with the Bernstein filtration of $W(C)$, that is, $\operatorname{det}_{w}$ is a homomorphism of multiplicative groups,

$$
\operatorname{det}_{w}: M_{N}(W(C)) \longrightarrow \overline{C[x, \xi]} .
$$

Let $P(x, D) \in M_{N}(W(C))$, and consider the mapping,

$$
P: \mathscr{A}_{R}^{N} \longrightarrow \mathscr{A}_{R}^{N} \quad(0<R \leq \infty) .
$$

Then for this mapping, we have the following index theorem, which is the most important in this paper.

Theorem 1 (Index theorem). Let $P(x, D) \in M_{N}(W(C))$ satisfy

$$
\operatorname{det}_{w}(P) \equiv p(x, \xi)=\sum_{m+n=l} a_{m n} x^{m} \xi^{n} \neq 0 .
$$

Then the mapping (1.6) has finite dimensional kernel and cokernel, and its index $\chi\left(P ; \hookrightarrow \mathscr{A}_{R}^{N}\right)\left(:=\operatorname{dim}_{C} \operatorname{Ker}\left(P ; \hookrightarrow \mathscr{A}_{R}^{N}\right)-\operatorname{codim}_{C} \operatorname{Im}\left(P ; \hookrightarrow \mathscr{A}_{R}^{N}\right)\right)$ is given by 


$$
\chi\left(P ; \hookrightarrow \mathscr{A}_{R}^{N}\right)=l-2 \sum_{t_{0} \in D_{R}} \operatorname{ord}_{t_{0}} p(2 t, 1),
$$

where $\operatorname{ord}_{t_{0}} p(2 t, 1)$ denotes the order of zeros of $p(2 t, 1)$ at $t_{0}$. Moreover, if $p(2 t, 1) \neq 0$ in $D_{R}$, then (1.6) is surjective.

Remark 1.1. If $\operatorname{det}_{w}(P) \equiv 0$ in the above theorem, we can prove that the mapping (1.6) has infinite dimensional kernel and cokernel, by the same way as in Miyake [10].

Since, $\mathscr{A}_{R}$ is an inductive sequence of topological spaces when $R \downarrow 0$ by the natural injection $\mathscr{A}_{R} \subset \mathscr{A}_{R^{\prime}}$ (densely) for $0<R^{\prime}<R \leq \infty$, we can define an inductive limit $\mathscr{A}$ of $\left\{\mathscr{A}_{R}\right\}_{R>0}$,

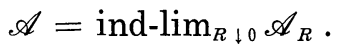

Then we have the following existence theorem.

Corollary (Existence). Under the assumption (1.7), the mapping

$$
P: \mathscr{A}^{N} \longrightarrow \mathscr{A}^{N}
$$

is surjective, and $\operatorname{dim}_{C} \operatorname{Ker}\left(P ; \hookrightarrow \mathscr{A}^{N}\right)=l$.

To state a result of regularity of solutions, we introduce a notion of regular trace following Aronszajn [4]. A trace $f \in \mathscr{A}_{R}$ is called a regular trace if the defining function $\tilde{f}(z, t) \in \operatorname{Sol}(H)_{R}$ of $f$, which is defined by the isomorphism (1.2), can be extended holomorphically at $t=0$. Moreover, $f \in \mathscr{A}$ is called a regular trace if it is represented by a regular trace in $\mathscr{A}_{R}$ for some $R>0$. Now, we have the following,

Theorem 2 (Regularity). Let $P(x, D) \in M_{N}(W(C))$ satisfy the assumption (1.7). Then the following statements are equivalent:

(a) Every $u \in \mathscr{A}^{N}$ such that $P u$ is regular trace is regular.

(b) $p(0,1) \neq 0$.

It will be useful to compare our results with those in the space of hyperfunctions, which we can not find in any references in our general form (see Komatsu [8]).

For a matrix $P(x, D) \in M_{N}(W(C))$, we denote by $\operatorname{det}_{\sigma}(P)$ the determinant of $P$ associated with the filtration of $W(C)$ by the order of differentiation, that is, $\operatorname{det}_{\sigma}$ is a homomorphism of multiplicative groups,

$$
\operatorname{det}_{\sigma}: M_{N}(W(C)) \longrightarrow\left\{a(x) \xi^{m} ; a(x) \in C[x], m=0,1,2, \cdots\right\},
$$

where $\boldsymbol{C}[x]$ denotes the set of polynomials in $x$. 
We denote by $\mathscr{B}(\Omega)$ the set of hyperfunctions on an open interval $\Omega \subset \boldsymbol{R}$. Let $P(x, D) \in M_{N}(W(C))$, and consider the mapping

$$
P(x, D): \mathscr{B}^{N}(\Omega) \longrightarrow \mathscr{B}^{N}(\Omega) .
$$

Then we have the following,

Theorem 1' (Existence). For a matrix $P(x, D)$, we assume

$$
\operatorname{det}_{\sigma}(P)=a(x) \xi^{m} \not \equiv 0 .
$$

Then the mapping (1.11) is surjective, and it holds that

$$
\operatorname{dim}_{\boldsymbol{C}} \operatorname{Ker}\left(P ; \hookrightarrow \mathscr{B}^{N}(\Omega)\right)=m+\sum_{x_{0} \in \Omega} \operatorname{ord}_{x_{0}} a(x) .
$$

THEOREM 2' (Regularity). Under the assumption (1.12), the following statements are equivalent:

(a) Every $u \in \operatorname{Ker}\left(P ; \hookrightarrow \mathscr{B}^{N}(\Omega)\right)$ is real analytic in $\Omega$.

(b) Every $u \in \mathscr{B}^{N}(\Omega)$ ) such that $P u$ is real analytic in $\Omega$ is real analytic in $\Omega$.

(c) $a(x) \neq 0$ in $\Omega$.

Remark 1.2. In Theorem 2, it is obvious that the condition that $p(0,1) \neq 0$ implies that every element in $\operatorname{Ker}\left(P ; \hookrightarrow \mathscr{A}^{N}\right)$ is regular trace. However, we do not know whether the converse does hold or not. We only note that the idea of the proof of Theorem 2 is rather similar to that of the equivalence of (a) and (c) in Theorem $2^{\prime}$, which is an essentia] part in the theorem.

\section{$\S 2$. Reduction of the problem and the proofs of theorems}

The following observation by Baouendi [5] makes it easy to study the differential equations in the space of traces.

For a couple $\left(\tilde{u}_{0}(t), \tilde{u}_{1}(t)\right) \in \mathscr{H}\left(D_{R}\right)^{2}$ we define $\tilde{u}(z, t)$ by

$$
\tilde{u}(z, t)=\sum_{l=0}^{\infty} \frac{z^{2 l}}{(2 l) !} D_{t}^{l} \tilde{u}_{0}(t)+\sum_{l=0}^{\infty} \frac{z^{2 l+1}}{(2 l+1) !} D_{t}^{l} \tilde{u}_{1}(t)
$$

Then it is easily seen that the right hand side converges uniformly on every compact set in $C_{z} \times D_{R}$, and hence $\tilde{u}(z, t) \in \operatorname{Sol}(H)_{R}$. Therefore the following isomorphism is obtained.

$$
\begin{aligned}
c: \operatorname{Sol}(H)_{R} & \longrightarrow \mathscr{H}\left(D_{R}\right)^{2} \\
\Psi & \\
\tilde{u}(z, t) & \longmapsto\left(\tilde{u}_{0}(t), \tilde{u}_{1}(t)\right)=\left(\tilde{u}(0, t), D_{z} \tilde{u}(0, t)\right)
\end{aligned}
$$


Thus we have a commutative diagram of isomorphisms,

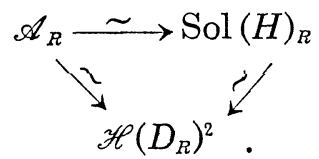

Let $P(x, D) \in W(C)$ and $u \in \mathscr{A}_{R}$. Then the defining function $\widetilde{P u}(z, t)$ $\in \operatorname{Sol}(H)_{R}$ of $P u$ is given by

$$
\widetilde{P u}(z, t)=\tilde{P}\left(z, t ; D_{z}\right) \tilde{u}(z, t),
$$

where

$$
\begin{array}{ll}
\tilde{P}\left(z, t ; D_{z}\right)=\sum_{k=0}^{\infty} \frac{t^{k}}{k !} P_{k}\left(z, D_{z}\right), & P_{0}\left(z, D_{z}\right)=P\left(z, D_{z}\right), \\
P_{k}\left(z, D_{z}\right)=\left[D_{z}^{2}, P_{k-1}\left(z, D_{z}\right)\right] & (k=1,2,3, \cdots),
\end{array}
$$

where $[A, B]:=A B-B A$ is the commutator of $A$ and $B$. To see this expression of $\tilde{P}\left(z, t ; D_{z}\right)$, it is sufficient to notice that $\mathscr{S}^{\prime}(\boldsymbol{R})$ is dense in $\mathscr{A}_{R}$ and the formula (0.1). It is also important that $\tilde{P}\left(z, t ; D_{z}\right)$ commutes with the heat operator $H=D_{t}-D_{z}^{2}$.

Thus, an equation $P u=f$ in $\mathscr{A}_{R}$ is translated to an equivalent one in $\operatorname{Sol}(H)_{R}$ for defining functions $\tilde{u}(z, t)$ and $\tilde{f}(z, t)$,

$$
\tilde{P}\left(z, t ; D_{z}\right) \tilde{u}(z, t)=\tilde{f}(z, t) \quad \text { in } \operatorname{Sol}(H)_{R} .
$$

Next, substitute the expressions (2.1) for $\tilde{u}$ and $\tilde{f}$ in the above equation, and compare the coefficients of $z^{0}$ and $z^{1}$ in both hand sides. Then we obtain an equivalent system of equations in $\mathscr{H}\left(D_{R}\right)^{2}$ for functions $\left(\tilde{u}_{0}, \tilde{u}_{1}\right)=\left(\tilde{u}(0, t), D_{z} \tilde{u}(0, t)\right)$ and $\left(\tilde{f}_{0}, \tilde{f}_{1}\right)=\left(\tilde{f}(0, t), D_{z} \tilde{f}(0, t)\right)$, which we express by

$$
\mathscr{P}\left(t, D_{t}\right)\left(\begin{array}{c}
\tilde{u}_{0} \\
\tilde{u}_{1}
\end{array}\right)=\left(\begin{array}{c}
\tilde{f}_{0} \\
\tilde{f}_{1}
\end{array}\right), \quad \mathscr{P}\left(t, D_{t}\right) \in M_{2}(W(C))
$$

We note that the equivalence of (2.5) with (2.4) is proved by the commutativity of $\tilde{P}\left(z, t ; D_{z}\right)$ with the heat operator and the uniqueness of the Cauchy problem for the heat equation in the $z$ direction.

For a matrix $P(x, D)=\left(P_{i j}\right) \in M_{N}(W(C))$ we consider the mapping,

$$
P: \mathscr{A}_{R}^{N} \longrightarrow \mathscr{A}_{R}^{N} \text {. }
$$

Then by the above argument, we know that this mapping is equivalent 
to the following one.

$$
\mathscr{P}: \mathscr{H}\left(D_{R}\right)^{2 N} \longrightarrow \mathscr{H}\left(D_{R}\right)^{2 N},
$$

where $\mathscr{P} \equiv\left(\mathscr{P}_{i j}\left(t, D_{t}\right)\right) \in M_{2 N}(W(C))$, and $\mathscr{P}_{i j} \in M_{2}(W(C))$ is defined from $P_{i j}$ as in the formula (2.5). Therefore, Theorem 1 is equivalent to the following,

Proposition 2.1. Under the assumption in Theorem 1, the mapping (2.7) has a finite index $\chi\left(\mathscr{P} ; \hookrightarrow \hookrightarrow \mathscr{H}\left(D_{R}\right)^{2 N}\right)=l-2 \sum_{t_{0} \in D_{R}} \operatorname{ord}_{t_{0}} p(2 t, 1)$. Moreover, if $p(2 t, 1) \neq 0$ in $D_{R}$, then $(2.7)$ is surjective.

On the other hand, this proposition is an immediate consequence of the following theorem.

Theorem 2.2 (Miyake [9]). Let $\Omega$ be a simply connected domain in $C$, and $P(x, D)$ be an $N \times N$ matrix of differential operators with holomorphic coefficients in $\Omega$. Let assume $\operatorname{det}_{\sigma}(P)=a(x) \xi^{m} \neq \equiv$, and zero points in $\Omega$ of $a(x)$ be finite. Then the mapping

$$
P: \mathscr{H}(\Omega)^{N} \longrightarrow \mathscr{H}(\Omega)^{N},
$$

has a finite index $\chi\left(P ; \hookrightarrow \mathscr{H}(\Omega)^{N}\right)=m-\sum_{x_{0} \in \Omega} \operatorname{ord}_{x_{0}} a(x)$. Moreover, if $a(x) \neq 0$ in $\Omega$, then the above mapping is surjective.

Therefore, to prove Theorem 1, it is sufficient to show the following,

Proposition 2.3. Let $P(x, D) \in M_{N}(W(C))$ and $\operatorname{det}_{w}(P) \equiv p(x, \xi)=$ $\sum_{m+n=l} a_{m n} x^{m} \xi^{n}$. Let $\mathscr{P}\left(t, D_{t}\right) \in M_{2 N}(W(C))$ be a matrix defined from $P$ by (2.7). Then it holds that

$$
\operatorname{det}_{\sigma}(\mathscr{P})=(p(2 t, 1))^{2}(-\tau)^{l} .
$$

We note that $(2.8)$ holds even when $p(x, \xi) \equiv 0$.

Therefore, our main purpose in the following is to prove (2.8), and it will be proved in Section 5 .

We remark here that there is no relations between $\operatorname{det}_{w}(P)$ and $\operatorname{det}_{\sigma}(P)$ in general, except the trivial one that $\operatorname{det}_{w}(P) \equiv 0$ if and only if $\operatorname{det}_{\sigma}(P) \equiv 0$. However, in a special case we can prove the following,

Proposition 2.4. Let $P(x, D) \in M_{N}(W(C))$ and put $\operatorname{det}_{w}(P)=p(x, \xi)$. If $p(0,1) \neq 0$, then $\operatorname{det}_{\sigma}(P)=p(0, \xi)$.

We give, here, the proofs of results by assuming above results. 
Proof of Corollary (Existence). Let $P(x, D) \in M_{N}(W(C))$ and put $\operatorname{det}_{w}(P)$ $=p(x, \xi)$. The assumption that $p(x, \xi) \not \equiv 0$ implies that $p(x, 1) \not \equiv 0$, since $p(x, \xi) \in \overline{C[x, \xi]}$. Hence for a sufficiently small $R_{0}>0$, it holds that $p(2 t, 1) \neq 0$ in $D_{R_{0}}$. Therefore, by Theorem 1 , for any $R$ such that $0<R \leq R_{0}$, the mapping $P: \mathscr{A}_{R}^{N} \rightarrow \mathscr{A}_{R}^{N}$ is surjective, and it holds that $\operatorname{dim}_{C} \operatorname{Ker}\left(P ; \hookrightarrow \mathscr{A}_{R}^{N}\right)=l$ (see (1.7)). Therefore corollary is obvious, since $\mathscr{A}$ is an inductive limit of $\left\{\mathscr{A}_{R}\right\}_{R>0}$.

Proof of Theorem 2 (Regularity). For $P(x, D) \in M_{N}(W(C))$ and $u \in \mathscr{A}_{R}^{N}$, we put $P u=f \in \mathscr{A}_{R}{ }^{N}$. Let $\tilde{u}(z, t)={ }^{t}\left(\tilde{u}_{1}, \cdots, \tilde{u}_{N}\right) \in \operatorname{Sol}(H)_{R}{ }^{N}$ and $\tilde{f}(z, t)=$ ${ }^{t}\left(\tilde{f}_{1}, \cdots, \tilde{f}_{N}\right) \in \operatorname{Sol}(H)_{R}^{N}$ be vectors of defining functions of components of $u$ and $f$, respectively. Here, ${ }^{t}(\cdots)$ denotes the transposed vector of $(\cdots)$. We put $\tilde{U}_{i}(t)=\left(\tilde{u}_{i}(0, t), D_{z} \tilde{u}_{i}(0, t)\right)(i=1, \cdots, N)$ and put $\tilde{U}(t)={ }^{t}\left(\tilde{U}_{1}(t)\right.$, $\left.\cdots, \tilde{U}_{N}(t)\right) \in \mathscr{H}\left(D_{R}\right)^{2 N}$. Let $\tilde{F}(t) \in \mathscr{H}\left(D_{R}\right)^{2 N}$ be a vector defined similarly from $f$. Then the system of equations $P u=f$ is equivalent to

$$
\mathscr{P}\left(t, D_{t}\right) \tilde{U}(t)=\tilde{F}(t),
$$

where $\mathscr{P}\left(t, D_{t}\right) \in M_{2 N}(W(C))$ is the one defined by (2.7).

From the assumption that $\operatorname{det}_{w}(P)=p(x, \xi) \not \equiv 0$, we can choose small $R_{0}$ such that for any $0<R \leq R_{0}$ the mapping $P: \mathscr{A}_{R}^{N} \rightarrow \mathscr{A}_{R}^{N}$ is surjective, and hence the mapping $\mathscr{P}: \mathscr{H}\left(D_{R}\right)^{2 N} \rightarrow \mathscr{H}\left(D_{R}\right)^{2 N}$ is also surjective. We note that for $\operatorname{det}_{\sigma}(\mathscr{P})=b(t) \tau^{\imath}$ (see Proposition 2.3), we may assume $b(t) \neq 0$ in $D_{R_{0}}$.

We remark here that the trace $u \in \mathscr{A}_{R}^{N}$ is regular if and only if $\tilde{U}(t)$ $\in \mathscr{H}\left(D_{R}\right)^{2 N}$ can be extended holomorphically at $t=0$, in view of the formula (2.1).

Under these preparations, we know that it is sufficient to show the equivalence of the following statements:

(a') Every $\tilde{U}(t) \in \mathscr{H}\left(D_{R}\right)^{2 N}$ such that $\mathscr{P} \tilde{U}$ can be extended holomorphically at $t=0$ can also be extended holomorphically at $t=0$.

$\left(b^{\prime}\right) \quad p(0,1) \neq 0$, i.e., $b(0) \neq 0$, where $0<R \leq R_{0}$.

It is almost evident that $\left(b^{\prime}\right)$ implies $\left(a^{\prime}\right)$, in view of Theorem 2.2. To show that $\left(\mathrm{a}^{\prime}\right)$ implies $\left(\mathrm{b}^{\prime}\right)$, let assume $b(0)=0$. Then we can take a simply connected open set $V$ including $D_{R} \cup\{0\}$ so that $b(t) \neq 0$ in $V \backslash\{0\}$. By Theorem 2.2, it holds that $\operatorname{dim}_{C} \operatorname{Ker}\left(\mathscr{P} ; \hookrightarrow \mathscr{H}\left(D_{R}\right)^{2 N}\right)=l, \operatorname{codim}_{C} \operatorname{Im}(\mathscr{P}$; $\left.\hookrightarrow \mathscr{H}\left(D_{R}\right)^{2 N}\right)=0$ and $\chi\left(\mathscr{P} ; \hookrightarrow \mathscr{H}(V)^{2 N}\right)<l$. Therefore, if the mapping 
$\mathscr{P}: \mathscr{H}(V)^{2 N} \rightarrow \mathscr{H}(V)^{2 N}$ is not surjective, there exists an element $\tilde{F}(t) \in \mathscr{H}(V)^{2 N}$ such that $\mathscr{P} \tilde{U}(t)=\tilde{F}(t)$ for some $\tilde{U}(t) \in \mathscr{H}\left(D_{R}\right)^{2 N} \backslash \mathscr{H}(V)^{2 N}$. On the other hand, if it is surjective, there is an element in $\operatorname{Ker}\left(\mathscr{P} ; \hookrightarrow \mathscr{H}\left(D_{R}\right)^{2 N}\right)$ which can not be extended at $t=0$ holomorphically, since $\operatorname{dim}_{C} \operatorname{Ker}\left(\mathscr{P} ; \hookrightarrow \mathscr{H}(V)^{2 N}\right)$ $=\chi\left(\mathscr{P} ; \hookrightarrow \hookrightarrow \mathscr{H}(V)^{2 N}\right)<l$. These facts contradict the statement $\left(\mathrm{a}^{\prime}\right)$.

We omit the proofs of Theorems $1^{\prime}$ and $2^{\prime}$, since they are done by the same way as in Komatsu [8] by using Theorem 2.2.

\section{§ 3. Calculation of the matrix $\mathscr{P}$ in (2.5)}

To calculate the matrix $\mathscr{P}$ for an operator $P(x, D) \in W(C)$, it is sufficient to consider an operator $P$ of monomial, in view of the linearity. Let

$$
P(x, D)=x^{m} D^{n}, \quad D=d / d x .
$$

We, first, calculate $\tilde{P}\left(z, t ; D_{z}\right)$ defined at (2.3). By the definition,

$$
\tilde{P}\left(z, t ; D_{z}\right)=\sum_{k=0}^{\infty} \frac{t^{k}}{k !} P_{k}\left(z, D_{z}\right)
$$

where $P_{0}\left(z, D_{z}\right)=P\left(z, D_{z}\right)=z^{m} D_{z}^{n}$ and $P_{k}\left(z, D_{z}\right)=D_{z}^{2} P_{k-1}-P_{k-1} D_{z}^{2}(k=1$, $2, \cdots)$. It is obvious that

$$
P_{1}\left(z, D_{z}\right)=2 m z^{m-1} D_{z}^{n+1}+m(m-1) z^{m-2} D_{z}^{n} .
$$

For general $k$, we set

$$
P_{k}\left(z, D_{z}\right)=\sum_{j=0}^{k} C_{k_{j}} \frac{m !}{(m-k-j) !} z^{m-k-j} D_{z}^{n+k-j}, \quad k>1 .
$$

Then we obtain the following recursion formula,

$$
C_{k 0}=2 C_{k-1,0}, \quad C_{k j}=C_{k-1, j-1}+2 C_{k-1, j}(1 \leq j \leq k-1), \quad C_{k k}=C_{k-1, k-1} .
$$

It is easily obtained that

$$
C_{k j}=2^{k-j} \frac{k !}{j !(k-j) !} .
$$

Hence we have

$$
\tilde{P}\left(z, t, D_{z}\right)=\sum_{k=0}^{m} \sum_{j=0}^{k} \frac{2^{k-j} \cdot m !}{j !(k-j) !(m-k-j) !} t^{k} z^{m-k-j} D_{z}^{n+k-j} .
$$

For $\tilde{u}(z, t) \in \operatorname{Sol}(H)_{R}$ we put its representation by 


$$
\tilde{u}(z, t)=\sum_{l=0}^{\infty} \frac{z^{2 l}}{(2 l) !} D_{t}^{l} \tilde{u}_{0}(t)+\sum_{l=0}^{\infty} \frac{z^{2 l+1}}{(2 l+1) !} D_{t}^{l} \tilde{u}_{1}(t)
$$

where $\tilde{u}_{0}(t), \quad \tilde{u}_{1}(t) \in \mathscr{H}\left(D_{R}\right)$. To calculate the coefficients of $z^{0}$ and $z^{1}$ of $\tilde{P}\left(z, t, D_{z}\right) \tilde{u}(z, t)$, we consider two cases as follows.

$\left.1^{\circ}\right)$ The case of $m+n=2 p(p=0,1,2, \cdots)$. The coefficient of $z^{0}$ is given by

$$
\sum_{j=0}^{[m / 2]} \frac{m !}{j !(m-2 j) !} \frac{(2 t)^{m-j}}{2^{j}} D_{t}^{p-j} \tilde{u}_{0}(t),
$$

where $[\cdot]$ denotes the Gauss symbol for real numbers. The coefficient of $z^{1}$ is calculated as follows.

$$
\begin{aligned}
\sum_{j=0}^{[m / 2]} \frac{m !}{j !(m-2 j) !} \frac{(2 t)^{m-j}}{2^{j}} D_{t}^{p-j} \tilde{u}_{1}(t) \\
\quad+\sum_{j=0}^{[(m-1) / 2]} \frac{m !}{j !(m-1-2 j) !} \frac{(2 t)^{m-1-j}}{2^{j}} D_{t}^{p-1-j} \tilde{u}_{1}(t) \\
=\quad \sum_{j=0}^{[(m+1) / 2]} \frac{(m+1) !}{j !(m+1-2 j) !} \frac{(2 t)^{m-j}}{2^{j}} D_{t}^{p-j} \tilde{u}_{1}(t) .
\end{aligned}
$$

Therefore, in this case we obtain

(3.6) $\mathscr{P}\left(t, D_{t}\right)=\left[\begin{array}{ll}\sum_{j=0}^{[m / 2]} \frac{m !}{j !(m-2 j) !} \frac{(2 t)^{m-j}}{2^{j}} D_{t}^{p-j}, & 0 \\ 0, & \sum_{j=0}^{[(m+1) / 2]} \frac{(m+1) !}{j !(m+1-2 j) !} \frac{(2 t)^{m-j}}{2^{j}} D_{t}^{p-j}\end{array}\right)=\mathscr{p u t} \mathscr{E}(m, n ; p)$.

$\left.2^{\circ}\right)$ The case of $m+n=2 q+1(q=0,1,2, \cdots)$. By the same way as the above, we obtain

(3.7) $\mathscr{P}\left(t, D_{t}\right)=\left[\begin{array}{ll}0, & \sum_{j=0}^{[m / 2]} \frac{m !}{j !(m-2 j) !} \frac{(2 t)^{m-j}}{2^{j}} D_{t}^{q-j} \\ \sum_{j=0}^{[(m+1) / 2]} \frac{(m+1) !}{j !(m+1-2 j) !} \frac{(2 t)^{m-j}}{2^{j}} D_{t}^{q+1-j}, & 0\end{array}\right] \underset{\text { put }}{=} \mathcal{O}(m, n ; q)$.

In special cases, we have the following correspondences:

$$
\begin{gathered}
D_{x} \longleftrightarrow\left(\begin{array}{ll}
0 & 1 \\
D_{t} & 0
\end{array}\right) \underset{\text { put }}{=} \mathscr{D}_{t}, \\
x \longleftrightarrow\left(\begin{array}{cr}
0 & 2 t \\
2 t D_{t}+1 & 0
\end{array}\right) \underset{\text { put }}{=} \mathscr{T} .
\end{gathered}
$$


These correspondences will play an important role in proving Proposition 2.3.

Summing up the above results, we obtain the following,

Proposition 3.1. Let $P(x, D) \in W(C)$ be

$$
P(x, D)=\sum_{l=0}^{p} \sum_{m+n=2 l} a_{m n} x^{m} D^{n}+\sum_{l=0}^{q} \sum_{m+n=2 l+1} b_{m n} x^{m} D^{n} .
$$

Then the matrix $\mathscr{P}\left(t, D_{t}\right) \in M_{2}(W(C))$ in (2.5) is given by

$$
\mathscr{P}\left(t, D_{t}\right)=\sum_{l=0}^{p} \sum_{m+n=2 l} a_{m n} \mathscr{E}(m, n ; l)+\sum_{l=0}^{q} \sum_{m+n=2 l+1} b_{m n} \mathcal{O}(m, n ; l) .
$$

Remark 3.2. In the case of $N=1$, Propositions 2.3 and 2.4 are obvious from above considerations and results in Section 4 . In fact,

1) If $p>q$, then $\operatorname{det}_{w}(P) \equiv p_{w}(x, \xi)=\sum_{m+n=2 p} a_{m n} x^{m} \xi^{n}$ and

$$
\operatorname{det}_{\sigma}(\mathscr{P})=\left\{\sum_{m+n=2 p} a_{m n}(2 t)^{m}\right\}^{2}(\tau)^{2 p}=\left(p_{w}(2 t, 1)\right)^{2}(-\tau)^{2 p} .
$$

Moreover, $p_{w}(0,1) \neq 0$ means $a_{0,2 p}=p_{w}(0,1) \neq 0$ and $a_{m, n}=0(m+n>2 p)$, and hence $\operatorname{det}_{\sigma}(P)=a_{0,2 p} \xi^{2 p}=p_{w}(0, \xi)$.

2) If $p \leq q$, then $\operatorname{det}_{w}(P) \equiv p_{w}(x, \xi)=\sum_{m+n=2 q+1} b_{m n} x^{m} \xi^{n}$ and

$$
\operatorname{det}_{\sigma}(\mathscr{P})=-\left\{\sum_{m+n=2 q+1} b_{m n}(2 t)^{m}\right\}^{2}(\tau)^{2 q+1}=\left(p_{w}(2 t, 1)\right)^{2}(-\tau)^{2 q+1} .
$$

As the above, we see that $p_{w}(0,1) \neq 0 \operatorname{implies}_{\operatorname{det}_{\sigma}}(P)=p_{w}(0, \xi)$.

\section{§4. Review of the determinant theory over the filtered rings}

We give here a brief summary of the determinant theory over the filtered rings or the ring of differential operators, since it seems to be not familiar to the researchers of differential equations.

Let $R$ be a filtered ring with filtration $F_{j} R(j=0,1,2, \cdots)$, which we denote by $(R, F)$. Here, $R$ is a non commutative unitary ring without zero divisors. That is, $F_{j} R(j=0,1,2, \cdots)$ are additive groups satisfying,

$$
\left\{\begin{array}{l}
R=\bigcup_{j=0}^{\infty} F_{j} R, \quad F_{0} R \subset F_{1} R \subset F_{2} R \subset \cdots, \\
F_{j} R \cdot F_{k} R \subset F_{j+k} R(j, k=0,1, \cdots) \quad \text { and } \quad F_{0} R \ni 1 .
\end{array}\right.
$$

We, further, assume the following relation,

$$
\left[F_{j} R, F_{k} R\right] \subset F_{j+k-1} R,
$$

where $[a, b]=a b-b a$. Let $\operatorname{gr}^{F} R$ be the graded ring associated with the filtration $F$, that is, 


$$
\operatorname{gr}^{F} R:=\bigoplus_{j=0}^{\infty} \bar{R}_{j}, \quad \bar{R}_{j}:=F_{j} R / F_{j-1} R .
$$

Then, obviously, $\operatorname{gr}^{F} R$ is a commutative ring.

Moreover, we assume that $R$ satisfies the so called left Ore property, that is, every finite non trivial left ideals have non empty intersection, or equivalently every non zero two elements have left non zero common multiples. Then, $R$ is embedded in a non commutative field $K$ canonically by constructing left quotients of $R$. Let $K^{\times}:=K \backslash\{0\}$ be the muliplicative group of $K, C$ be a commutator subgroup of $K^{\times}$and $\bar{K}:=K^{\times} / C$. Then, by Dieudonné [7], for matrices in $M_{N}(R)$ the determinant, which we denote by $\Delta$, is defined as a homomorphism of multiplicative groups,

$$
\Delta: M_{N}(R) \longrightarrow \bar{K} \cup\{0\},
$$

which is a natural extension of the canonical map $\Delta: R \rightarrow \bar{K} \cup\{0\}$ (see Dieudonné [7] or Miyake [9] for detail).

In our purpose, we need further consideration. Let

$$
\sigma_{F}: R \longrightarrow \operatorname{gr}^{F} R
$$

be the canonical homomorphism, and call it the symbol map. Then the symbol map (4.5) induces naturally the symbol map

$$
\sigma_{F}: K \longrightarrow \operatorname{gr}^{F} K
$$

by $\sigma_{F}\left(a^{-1} \cdot b\right):=\sigma_{F}(a)^{-1} \cdot \sigma_{F}(b)$ for an element $a^{-1} \cdot b \in K(a, b \in R)$. Here, $\mathrm{gr}^{F} K$ is a field of quotients of commutative ring $\mathrm{gr}^{F} R$. It is obvious that $\sigma_{F}(a)=\sigma_{F}(b)$ for any $a, b \in K^{\times}$such that $a=b(\bmod C)$.

Now, the determinant for matrices in $M_{N}(R)$ associated with the filtration $F$, which we denote by $\operatorname{det}_{F}$, is $\operatorname{defined}$ by $\operatorname{det}_{F}:=\sigma_{F} \circ \Delta$. Thus, $\operatorname{det}_{F}$ is defined as a homomorphism of multiplicative groups,

$$
\operatorname{det}_{F}: M_{N}(R) \longrightarrow \operatorname{gr}^{F} K \text {. }
$$

We remark that when $N=1$ the determinant of an element $a \in R$ is so defined that $\operatorname{det}_{F}(a)=\sigma_{F}(a) \in \operatorname{gr}^{F} R$.

The following result due to Adjamagbo is fundamental.

Proposition 4.1 (Adjamagbo [1]). If $\mathrm{gr}^{F} R$ is a unique factorization ring, then the determinant $\operatorname{det}_{F}$ is $\operatorname{gr}^{F} R$ valued.

It was already shown that the ring of (partial) differential operators 
with holomorphic coefficients satisfies the left and right Ore properties, and is also for the Weyl algebra of any dimension (see Björk [6] and Schapira [12]). In our case, the proof is easily done by the Euclidean algorithm for ordinary differential operators.

The Bernstein filtration of the Weyl algebra is given by $F_{j}(W(\boldsymbol{C}))$ : = $\left\{P=\sum_{m+n \leq j} a_{m n} x^{m} D^{n}\right\}(j=0,1,2, \cdots)$, and its graded ring is nothing but $\bar{C}[x, \xi]$. Hence, in this case the determinant, which we denote by $\operatorname{det}_{w}$, is a homomorphism of multiplicative groups,

$$
\operatorname{det}_{w}: M_{N}(W(C)) \longrightarrow \overline{C[x, \xi]} .
$$

Let $\mathscr{D}(\Omega)$ denote the set of differential operators with holomorphic coefficients in a domain $\Omega \subset C$. Then the natural filtration of $\mathscr{D}(\Omega)$ by the order of differentiation $F_{j} \mathscr{D}(\Omega):=\left\{P(x, D)=\sum_{m \leq j} a_{m}(x) D^{m} ; a_{m}(x) \epsilon\right.$ $\mathscr{H}(\Omega)\}(j=0,1,2, \cdots)$ induces the determinant $\operatorname{det}_{\sigma}$ for matrices in $M_{N}(\mathscr{D}(\Omega))$ as a homomorphism of multiplicative groups,

$$
\operatorname{det}_{\sigma}: M_{N}(\mathscr{D}(\Omega)) \longrightarrow\left\{a(x) \xi^{m} ; a(x) \in \mathscr{H}(\Omega), m=0,1,2, \cdots\right\}
$$

(see Miyake [9], and Sato and Kashiwara [11] for general case of dimension).

We give here fundamental properties of the determinant.

For an element $a \in R$ the order of $a, \operatorname{ord}_{F}(a)$, is defined by

$$
\operatorname{ord}_{F}(a)= \begin{cases}\min \left\{j ; a \in F_{j} R\right\} & \text { if } a \neq 0 \\ -\infty & \text { if } a=0\end{cases}
$$

That is, $\operatorname{ord}_{F}(a)=\operatorname{deg}\left(\sigma_{F}(a)\right)$, where $\operatorname{deg}\left(\sigma_{F}(a)\right)$ denotes the degree of $\sigma_{F}(a)$ in $\operatorname{gr}^{F} R$.

Let $A=\left(a_{i_{j}}\right) \in M_{N}(R)$ and put $m_{i_{j}}=\operatorname{ord}_{F}\left(a_{i_{j}}\right)$. Then the total order of $A, \operatorname{ord}_{F}(A)$, is defined by

$$
\operatorname{ord}_{F}(A)=\max _{\pi \in \Phi_{N}} \sum_{i=1}^{N} m_{i \pi(i)} \in\{-\infty\} \cup\{0,1,2,3, \cdots\},
$$

where $\widetilde{\Im}_{N}$ denotes the permutation group of $\{1,2, \cdots, N\}$ and we define $-\infty+l=-\infty$ for any $l$.

Let $\operatorname{ord}_{F}(A)=m \geq 0$. Then, by Volevič's lemma (cf. Miyake [9]) there is a system of integers $\left\{s_{i}, t_{j}\right\}$ such that

$$
m_{i j} \leq t_{j}-s_{i} \text { and } m=\sum t_{j}-\sum s_{i} .
$$

Moreover, $A$ is said to be non degenerate if

$$
\text { degree of } \operatorname{det}\left(\sigma_{F}\left(a_{i_{j}}\right)\right)=m .
$$


We note that $\operatorname{det}\left(\sigma_{F}\left(a_{i j}\right)\right) \in \operatorname{gr}^{F} R$ is well defined, since $\operatorname{gr}^{F} R$ is commutative.

Now we have

TheOREM 4.2. We assume that $\operatorname{gr}^{F} R$ is a unique factorization ring. Then we have:

(a)

$$
\operatorname{det}_{F}\left(\text { Triang }\left\{A_{1}, \cdots, A_{k}\right\}\right)=\prod_{j=1}^{k} \operatorname{det}_{F}\left(A_{j}\right),
$$

where Triang $\left\{A_{1}, \cdots, A_{k}\right\}$ denotes the block wise triangular matrix with the $j$-th diagonal block $A_{j} \in M_{N j}(R)(j=1,2, \cdots, k)$.

(b) Let $A=\left(a_{i_{j}}\right) \in M_{N}(R)$ be of $\operatorname{ord}_{F}(A)=m \geq 0$. Then $A$ is non degenerate if and only if

$$
\operatorname{det}_{F}(A)=\text { the homogeneous part of degree } m \text { of } \operatorname{det}\left(\sigma_{F}\left(a_{i_{j}}\right)\right) \text {. }
$$

Moreover, in this case, we have

where

$$
\operatorname{det}_{F}(A)=\operatorname{det}\left(\circ_{F}\left(a_{i j}\right)\right)
$$

$$
\stackrel{\circ}{\sigma}_{F}\left(a_{i j}\right)=\left\{\begin{array}{lll}
\sigma_{F}\left(a_{i j}\right) & \text { if } & m_{i j}=t_{j}-s_{i} \\
0 & \text { if } & m_{i j}<t_{j}-s_{i}
\end{array} .\right.
$$

(c) If $\operatorname{ord}(A)=-\infty$, then $\operatorname{det}_{F}(A)=0$. Conversely, if $\operatorname{det}_{F}(A)=0$, then there is a matrix $B$ with $\operatorname{det}_{F}(B) \neq 0$ such that $\operatorname{ord}_{F}(B A)=-\infty$ (or $\left.\operatorname{ord}_{F}(A B)=-\infty\right)$.

\section{§ 5. Proofs of Propositions 2.3 and 2.4}

Let $\mathscr{W}(C)\left(\subset M_{2}(W(C))\right.$ be an algebra over $C$ generated by $\mathscr{D}_{t}$ and $\mathscr{T}$, where $\mathscr{D}_{t}$ and $\mathscr{T}$ are matrices defined by (3.8) and (3.9) respectively.

The following obvious relation

$$
\left[\mathscr{D}_{t}, \mathscr{T}\right] \equiv \mathscr{D}_{t} \mathscr{T}-\mathscr{T} \mathscr{D}_{t}=I_{2} \text { (the identity matrix of size 2), }
$$

implies immediately the following,

LEMMA 5.1. The correspondences (3.8) and (3.9) induce an isomorphism of $W(\boldsymbol{C})$ and $\mathscr{W}(\boldsymbol{C})$, and we denote this isomorphism by

$$
\varphi: W(\boldsymbol{C}) \longrightarrow \mathscr{W}(\boldsymbol{C}) \text {. }
$$

Lemma 5.2. For a matrix $P(x, D) \in W(C)$, let $\mathscr{P}\left(t, D_{t}\right) \in M_{2}(W(C))$ be the one defined by (3.10). Then it holds that $\varphi(P) \equiv P\left(\mathscr{T}, \mathscr{D}_{t}\right)=\mathscr{P}\left(t, D_{t}\right)$.

Proof. It is sufficient to prove $\mathscr{T}^{m} \mathscr{D}_{t}^{2 p-m}=\mathscr{E}(m, 2 p-m ; p)$ and 
$\mathscr{T}^{m} \mathscr{D}_{t}^{2 q+1-m}=\mathcal{O}(m, 2 q+1-m ; q)$ respectively. We shall prove only the former equality, since another one is proved similarly.

We put $m+n=k$ and consider $x^{m} D^{n}$. When $k=1$, there is nothing to prove. We assume the equalities are proved up to $k<2 p$, and consider the case $k=2 p$. When $m=0$ and 1 , it is more easy, so we assume $m \geq 2$. The right hand side of the relation $\varphi\left(x^{m} D^{2 p-m}\right)=\varphi(x) \varphi\left(x^{m-1} D^{2 p-m}\right)$ is written by the induction assumption as

$$
\begin{aligned}
& \left(\begin{array}{cc}
0 & 2 t \\
2 t D_{t}+1 & 0
\end{array}\right)\left(\begin{array}{ccc}
0, & \sum_{j=0}^{[(m-1) / 2]} \frac{(m-1) !}{j !(m-1-2 j) !} \frac{(2 t)^{m-1-j}}{2^{j}} D_{t}^{p-1-j} \\
\sum_{j=0}^{[m / 2]} \frac{m !}{j !(m-2 j) !} \frac{(2 t)^{m-1-j}}{2^{j}} D_{t}^{p-j}, & 0
\end{array}\right) \\
& \underset{\text { put }}{=}\left(\begin{array}{ll}
P_{11} & P_{12} \\
P_{21} & P_{22}
\end{array}\right) .
\end{aligned}
$$

Therefore $P_{12}=P_{21}=0$, and

$$
\begin{aligned}
P_{11}= & \sum_{j=0}^{[m / 2]} \frac{m !}{j !(m-2 j) !} \frac{(2 t)^{m-j}}{2^{j}} D_{t}^{p-j}, \\
P_{22}= & (2 t D+1) \sum_{j=0}^{[(m-1) / 2]} \frac{(m-1) !}{j !(m-1-2 j) !} \frac{(2 t)^{m-1-j}}{2^{j}} D_{t}^{p-1-j} \\
= & \sum_{j=0}^{[(m-1) / 2]} \frac{(m-1) !}{j !(m-1-2 j) !} \frac{(2 t)^{m-j}}{2^{j}} D_{t}^{p-j} \\
& +\sum_{j=0}^{[(m-1) / 2]} \frac{(m-1) !}{j !(m-1-2 j) !}\{2(m-1-j)+1\} \frac{(2 t)^{m-1-j}}{2^{j}} D_{t}^{p-1-j} . \\
= & \sum_{j=0}^{[(m+1) / 2]} \frac{(m+1) !}{j !(m+1-2 j) !} \frac{(2 t)^{m-j}}{2^{j}} D_{t}^{p-j} .
\end{aligned}
$$

This completes the proof.

The isomorphism $\varphi$ defined by (5.2) induces an isomorphism of rings of matrices by,

$$
\begin{gathered}
\varphi: M_{N}(W(C)) \longrightarrow M_{N}(\mathscr{W}(\boldsymbol{C}))\left(\subset M_{2 N}(W(C))\right), \\
\stackrel{\Psi}{=}\left(P_{i j}\right) \longmapsto \varphi(P) \stackrel{\left(\varphi\left(P_{i j}\right)\right)}{\longrightarrow}
\end{gathered}
$$

where $\varphi(P)=\mathscr{P}\left(t, D_{t}\right)$ given in (2.7).

Proof of Proposition 2.3. A matrix $Q(x, D) \in M_{N}(W(C))$ is said to be of elementary type if 


$$
Q=\operatorname{Diag}\left\{1, \cdots, 1, \stackrel{j}{Q}_{j}, 1, \cdots, 1\right\} \quad \text { or } \quad Q=\operatorname{Triang}\{1, \cdots, 1\},
$$

where Diag $\left\{Q_{1}, \cdots, Q_{N}\right\}$ denotes the diagonal matrix with the $j$-th diagonal component $Q_{j}$.

For a matrix $Q(x, D)$ of elementary type,

$$
\operatorname{det}_{w}(Q) \equiv q(x, \xi)=\left\{\begin{array}{cl}
\sigma_{w}\left(Q_{j}\right) & \text { for the diagonal case } \\
1 & \text { for the triangular case }
\end{array}\right.
$$

We put $\operatorname{deg} q(x, \xi)=k(q(x, \xi) \in \overline{C[x, \xi]})$. Then by Remark 3.2 and Theorem 4.2 , it holds that

$$
\operatorname{det}_{\sigma}(\varphi(Q))=(q(2 t, 1))^{2}(-\tau)^{k}
$$

An effective method of calculation of determinant of a matrix $P(x, D)$ $\in M_{N}(W(C))$ is done as follows. In view of the left Ore property of $W(\boldsymbol{C})$, there are matrices $Q_{j}(x, D) \in M_{N}(W(C))(j=1, \cdots, k)$ of elementary type such that

$$
Q_{k} \cdots Q_{2} Q_{1} P=\text { Triang }\left\{R_{1}, \cdots, R_{N}\right\} \frac{\overline{\text { put }}}{\bar{~}} R(x, D), \quad R_{j} \in W(C) .
$$

This implies

$$
\varphi\left(Q_{k}\right) \cdots \varphi\left(Q_{1}\right) \varphi(P)=\text { Triang }\left\{\varphi\left(R_{1}\right), \cdots, \varphi\left(R_{N}\right)\right\}=\varphi(R) .
$$

We put

$$
\begin{aligned}
& \operatorname{det}_{w}\left(Q_{j}\right)=q_{\jmath}(x, \xi), \quad \operatorname{deg} q_{j}(x, \xi)=q_{j}, \\
& \operatorname{det}_{w}(R)=\prod_{j=1}^{N} \operatorname{det}_{w}\left(R_{j}\right) \frac{\overline{\text { put }}}{\ln } r(x, \xi), \quad \operatorname{deg} r(x, \xi)=r .
\end{aligned}
$$

Then we have

$$
\begin{aligned}
\operatorname{det}_{w}(P) & =\operatorname{det}_{w}(R) \cdot\left\{\prod_{j=1}^{k} \operatorname{det}_{w}\left(Q_{j}\right)\right\}^{-1}=r(x, \xi) \cdot\left\{\prod_{j=1}^{k} q_{j}(x, \xi)\right\}^{-1} \\
& \overline{\overline{\text { put }}} p(x, \xi), \quad \operatorname{deg} p(x, \xi) \equiv p=r-\sum_{j=1}^{k} q_{j} .
\end{aligned}
$$

Considering the relations (5.4) and (5.5), we obtain

$$
\begin{aligned}
\operatorname{det}_{\sigma}(\varphi(P)) & =\operatorname{det}_{\sigma}(\varphi(R)) \cdot\left\{\prod_{j=1}^{k} \operatorname{det}_{\sigma}\left(\varphi\left(Q_{j}\right)\right)\right\}^{-1} \\
& =(r(2 t, 1))^{2}(-\tau)^{r} \cdot\left\{\prod_{j=1}^{k}\left(q_{j}(2 t, 1)\right)^{2}(-\tau)^{q_{j}}\right\}^{-1}
\end{aligned}
$$




$$
\begin{aligned}
& =\left[r(2 t, 1) \cdot\left\{\sum_{j=1}^{k} q_{j}(2 t, 1)\right\}^{-1}\right]^{2} \cdot(-\tau)^{r-q_{1} \cdots-q_{k}} \\
& =(p(2 t, 1))^{2}(-\tau)^{p} .
\end{aligned}
$$

This completes the proof.

Proof of Proposition 2.4. For a matrix $P=\left(P_{i j}\right) \in M_{N}(W(C))$, we put $\operatorname{ord}_{w}\left(P_{i j}\right)=p_{i j}$ and $\operatorname{ord}_{D}\left(P_{i j}\right)=q_{i j}$. Also, we denote by $\operatorname{ord}_{w}(P)$ and $\operatorname{ord}_{D}(P)$ the total orders of $P$ with respect to the Bernstein filtration and the filtration by the order of differentiation respectively.

By the definition, it is obvious that $\operatorname{ord}_{w}(P) \geq \operatorname{ord}_{D}(P)$. Let $\operatorname{det}_{w}(P)$ $=p(x, \xi)$ and put $l=\operatorname{deg} p(x, \xi)$. Then $P(x, D)$ is non degenerate in the Bernstein filtration if and only if $\operatorname{ord}_{w}(P)=l$.

We shall prove our proposition dividing into two cases.

1) The case where $P$ is not degenerate in the Bernstein filtration.

We choose a system of integers $\left\{s_{i}, t_{j}\right\}$ such that

$$
p_{i j} \leq t_{j}-s_{i} \text { and } l=\operatorname{ord}_{w}(P)=\sum t_{j}-\sum s_{i} .
$$

We put

$$
\left\{\begin{array}{l}
\dot{P}_{i j}(x, \xi)=\dot{\sigma}_{w}\left(P_{i j}\right) \quad(\text { see Theorem } 4.2), \\
\dot{P}_{w}(x, \xi)=\left(\dot{P}_{i j}(x, \xi)\right) .
\end{array}\right.
$$

Then $p(x, \xi)=\operatorname{det} \stackrel{\circ}{P}_{w}(x, \xi)$. By the assumption that $p(0,1) \neq 0$, we have $\operatorname{det} \dot{P}_{w}(0, \xi)=p(0,1) \xi^{l}=p(0, \xi) \not \equiv 0$. This implies that $\operatorname{ord}_{D}(P) \geq \operatorname{ord}_{w}(P)$, and hence $\operatorname{ord}_{D}(P)=\operatorname{ord}_{w}(P)$. Moreover, this equality means that for any permutation $\pi \in \mathcal{S}_{N}$ such that $\sum_{i} q_{i \pi(i)}=l$, it holds that

$$
\sigma\left(P_{i \pi(i)}\right)=a_{i \pi(i)}(x) \xi^{q_{i(i)}} \text { and } a_{i \pi(i)}(x) \equiv \text { const } .
$$

These imply that $\operatorname{det}_{\sigma}(P)=\operatorname{det} \stackrel{\circ}{P}_{w}(0, \xi)=p(0, \xi)$, since $p_{i j} \geq q_{i j}$.

2) The case where $P$ is a degenerate matrix in the Bernstein filtration.

By the assumption, it holds that $\operatorname{ord}_{w}(P)>l=\operatorname{deg} p(x, \xi)$ and $\operatorname{det} \dot{P}_{w}(x, \xi) \equiv 0$. Therefore, there is a non zero left null vector $l(x, \xi)=$ $\left(l_{1}(x, \xi), \cdots, l_{N}(x, \xi)\right)$ of $\dot{P}_{w}(x, \xi)$ of relatively prime $l_{i}(x, \xi) \in C[x, \xi](j=1$, $\cdots, N)$. We may assume that there exist $i$ and $r$ such that

$$
l_{i}(x, \xi)=\xi^{r}+\sum_{k=1}^{r} c_{k} x^{k} \xi^{r-k}, \quad c_{k} \in \boldsymbol{C} .
$$

We put 


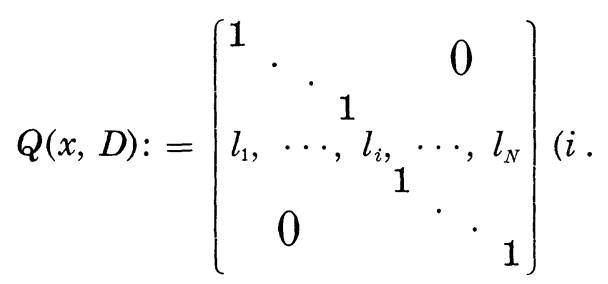

Then we have $\operatorname{det}_{w}(Q)=l_{i}(x, \xi), \operatorname{ord}_{w}(Q)=r$ and $\operatorname{ord}_{w}(Q P) \leq \operatorname{ord}_{w}(P)+$ $r-1$. If $Q P$ is degenerate in the Bernstein filtration, we continue this operation. Then we obtain matrices of above type $\left\{Q_{j}(x, D)\right\}_{j=1}^{k}$ such that

$$
\operatorname{ord}_{w}\left(Q_{k} \cdots Q_{1} P\right) \leq \operatorname{ord}_{w}(P)+\sum_{j=1}^{k} r_{j}-k
$$

where $r_{j}=\operatorname{ord}_{w}\left(Q_{j}\right)$. It should be remarked that the inequality $k \leq \operatorname{ord}_{w}(P)$ does hold. In fact, the following equality

$$
\operatorname{det}_{w}(P)=\operatorname{det}_{w}\left(P_{k}\right) \cdot\left\{\prod_{j=1}^{k} \operatorname{det}_{w}\left(Q_{j}\right)\right\}^{-1} \quad\left(P_{k}:=Q_{k} \cdots Q_{1} P\right)
$$

implies

$$
\operatorname{deg}\left(\operatorname{det}_{w}(P)\right)=\operatorname{deg}\left(\operatorname{det}_{w}\left(P_{k}\right)\right)-\sum_{j=1}^{k} \operatorname{deg}\left(\operatorname{det}_{w}\left(Q_{j}\right)\right) \leq \operatorname{ord}_{w}(P)-k .
$$

Therefore, $\operatorname{det}_{w}(P)=0$ if $k>\operatorname{ord}_{w}(P)$, which is a contradiction. Now, we may assume that $P_{k}$ is non degenerate in the Bernstein filtration. We note also that for matrices $Q_{j}(x, D)(j=1, \cdots, k)$ and $P_{k}(x, D)$, the assertion of the proposition is true. Hence, our assertion does hold for a matrix $P(x, D)$ in view of the relation,

$$
\operatorname{det}_{\sigma}(P)=\operatorname{det}_{\sigma}\left(P_{k}\right) \cdot\left\{\prod_{j=1}^{k} \operatorname{det}_{\sigma}\left(Q_{j}\right)\right\}^{-1} .
$$

Added in proof. The author has proved, recently, the converse of the statement in Remark 1.2 holds. Therefore, Theorem 2 corresponds completely to Theorem $2^{\prime}$. The detail will be given in the forthcoming paper.

\section{REFERENCES}

[1] Adjamagbo, K., Déterminant sur des anneaux filtrés, C.R. Acad. Sci. Paris, 293 (1981) , 447-449.

[2] - Théorèmes d'indece pour les systèmes généraux d'équations différentielles linéaires, Séminaire de J. Vaillant de Univ. Paris 6, (1982-83), 134-165. 
[ 3 ] Aronszajn, N., Preliminary notes for "Traces of analytic solutions of the heat equation”, Colloque International C.N.R.S. sur les équations aux dérivées partielles linéaire, Astérisque, 2-3 (1973), 5-34.

[4] - Traces of analytic solutions of the heat equation, ibid., 2-3 (1973), 35-68.

[5] Baouendi, M. S., Solvability of partial differential equations in the traces of analytic solutions of the heat equation, Amer. Jour. Math., 97 (1976), 983-1005.

[ 6 ] Björk, J. E., Rings of Differential Operators, North-Holland Publ. Co., AmsterdamOxford-New York, 1979.

[7] Dieudonné, J., Les déterminants sur un corp non commutatif, Bull. Soc. Math. France, 71 (1943), 27-45.

[ 8 ] Komatsu, H., Introduction to the theory of hyperfunctions (in Japanese), Iwanami Publ., Tokyo, 1978.

[9 ] Miyake, M., On the determinant of matrices of ordinary differential operators and an index theorem, Funk. Ekvac., 26 (1983), 155-171.

[10] - On the irregularity for general systems of differential equations in the complex domain, ibid., 26 (1983), 211-230.

[11] Sato, M. and M. Kashiwara, The determinant of pseudo-differential operators, Proc. Japan Acad., 51 (1975), 17-19.

[12] Schapira, P., Microdifferential systems in the complex domain, Springer-Verlag, Berlin-Heiderberg-New York-Tokyo, 1985.

Department of Mathematics

College of General Education

Nagoya University

Chikusa-ku, Nagoya, 464

Japan 